\title{
A Qualitative Investigation of the Interactions of Students with Graduate and Undergraduate TAs in General Chemistry Laboratories
}

\author{
Angela Thien Huong Nguyen ${ }^{1}$ D, Xavier D Antoine-Goeas ${ }^{1}$, Muhammad Sulman ${ }^{1}$, Linh Le Vuong Tra ${ }^{1}$, \\ Charles T Cox, Jr. ${ }^{2}$ and Ozcan Gulacar ${ }^{1, * \mathbb{D}}$ \\ 1 Department of Chemistry, University of California-Davis, One Shields Avenue, Davis, CA 95616, USA; \\ athnguy@ucdavis.edu (A.T.H.N.); xantoine@ucdavis.edu (X.D.A.-G.); msulman@ucdavis.edu (M.S.); \\ lvtra@ucdavis.edu (L.L.V.T.) \\ 2 Department of Chemistry, Duke University, 128 Science Drive, Durham, NC 27708, USA; \\ charlie.cox@duke.edu \\ * Correspondence: ogulacar@ucdavis.edu
}

\section{check for} updates

Citation: Nguyen, A.T.H.; Antoine-Goeas, X.D.; Sulman, M.; Tra, L.L.V.; Cox, C.T., Jr.; Gulacar, O. A Qualitative Investigation of the Interactions of Students with Graduate and Undergraduate TAs in General Chemistry Laboratories. Educ. Sci. 2021, 11, 655. https://doi.org/ 10.3390/educsci11100655

Academic Editors: Stephen A

Butterfield and Eila Jeronen

Received: 31 August 2021

Accepted: 14 October 2021

Published: 19 October 2021

Publisher's Note: MDPI stays neutral with regard to jurisdictional claims in published maps and institutional affiliations.

Copyright: (c) 2021 by the authors. Licensee MDPI, Basel, Switzerland. This article is an open access article distributed under the terms and conditions of the Creative Commons Attribution (CC BY) license (https:// creativecommons.org/licenses/by/ $4.0 /)$.

\begin{abstract}
A new teaching assistant model was adopted and qualitatively assessed for the general chemistry laboratory, in which both an undergraduate and a graduate teaching assistant provided instruction to students during the lab. Verbal interactions between graduate and undergraduate teaching assistants were recorded, transcribed, and coded using the modified Laboratory Observation Protocol for Undergraduate STEM (LOPUS). The codes were applied to capture how discussions, questioning, and explanations were conducted. In addition, the content discussed was coded to identify specific areas that pose challenges for students. Sizable differences were not observed between the number of interactions of the initiation, explanation, and questioning codes between graduate and undergraduate teaching assistants. Of the interactions, $77 \%$ focused on questions and discussions regarding the experimental setup. Discussions on the implications with regards to the effective use of undergraduate and graduate teaching assistants in chemistry laboratories are included.
\end{abstract}

Keywords: laboratory; peer-learning; teaching assistant

\section{Introduction}

Best practices for teaching science, technology, engineering, and math, collectively known as STEM, have been widely researched to improve students' performance, satisfaction, and retention in STEM courses and majors. New pedagogies have been developed using evidence-based research for improving lecture and laboratory instruction. Chemistry is a visual and hands-on science, which implies that much of the actual learning often occurs outside of the usual lecture classroom setting [1]. Recitations are incorporated with general and organic chemistry to supplement lectures and reinforce problem solving, and lab instruction is incorporated to provide effective hands-on experience for students to engage in the content [2]. Learning in the lab gives students a different perspective on the material by introducing ways to apply concepts in practice [3] and discover concepts. Because the laboratory component provides an additional layer for promoting learning and resonates strongly with various learning styles [4], instruction in laboratories has been proven to increase students' understanding of chemistry concepts [5].

In addition to introducing lab techniques and nurturing scientific skills, instruction received in the lab has great potential in promoting problem solving in small groups and fostering a positive learning environment [6]. Although labs are designed to encourage students to delve deeper into concepts, students have expressed that they are usually focused on learning facts, such as experimental procedures or properties of elements, 
rather than engaging in conceptual understanding [7]. Students emphasize good grades over in-depth learning, leading to greater focus on successfully completing experiments instead of fully understanding the conceptual basis for experiments [6]. Generally, students retain more information regarding the procedural aspect of the experiment rather than the underlying concepts, but the importance of knowing the conceptual aspect is greater than recounting facts [7].

Given the differences in teaching environments in labs versus lectures, there is a need to develop a different approach and utilize more unique ways to study the teaching methods and effectiveness of lab instruction [8]. However, due to various requirements and the limited constructs of study designs, many methods are not ideal to capture the natural learning environment of labs [9]. Although the benefits and value of learning in the lab are widespread, there are not many well-designed studies to support this belief [6]. The data collection methods, such as surveys and interviews, could make participants hesitant to act as they usually would under normal circumstances, creating an overly unnatural learning environment and leading to artificial results [9]. However, unobtrusive methods of collecting data, such as audio or video recording, will provide the most candid data that can be further analyzed without creating intrusive changes to the labs' usual proceedings [9].

Student learning within the lab is accomplished under the lead instruction of a graduate teaching assistant (GTA) $[4,10,11]$. In most lab sections, the GTA is the sole instructor, in charge of leading the class, supervising all experiments, and assessing students' understanding through various interactions related to the experiments [7]. As a part of their duties, GTAs are expected to give a brief lecture at the beginning of the labs to explain the experiment's underlying theoretical concepts, address safety practices, review the experiment's procedures, and emphasize the laboratory objectives $[4,7]$. Being the sole lab instructor, GTAs are an integral part of student learning within the lab and play a large role in stimulating students' interest in learning science [6]. Students' close interactions with GTAs improve their motivation and, in turn, spark an interest in STEM majors [3,9]. GTAs play a key role in maintaining students' interest and supporting students' interest by connecting abstract concepts and an invisible world to concrete products [4]. Therefore, GTAs not only support students' understanding of concepts behind the experiments, but also help students apply these principles to real-world issues [3]. Without proper guidance, students may lose interest in STEM subjects due to confusion regarding connections between experimental findings and real-world applications [3,9].

Although GTAs are responsible for all aspects of the experiments, the strong emphasis on safety in the scientific community and the limited lab timeframe results in GTAs worrying more about guiding students to safely finish their experiments rather than spending enough time with each group to encourage them to question the experiments' details $[6,12]$. Because of GTAs' instructional emphasis and limitations, students have expressed that they are usually focused on learning facts, such as experimental procedures or elemental properties, rather than engaging in conceptual understanding [7]. This focus results in a culture that encourages students to care more about completing experiments and getting good grades instead of pursuing in-depth learning to fully understand the conceptual basis of experiments [6]. For this reason, despite GTAs' good intentions, students usually end up retaining more procedural information rather than understanding scientific concepts and developing problem-solving skills essential for scientific advancement of individuals and societies [7].

In order to enhance students' learning in lectures and recitations, science educators have put forth a great effort and utilized different methods. Some of these methods utilized peer learning in the form of learning assistants (LAs), which are selected from among fellow undergraduates who previously completed the course successfully [13-15]. Several studies investigated the effectiveness of such programs and reported positive results. The reported benefits of introducing LAs include, but are not limited to, enhancing the inclusivity of the classroom [16], promoting an increase in student performance [15,17], improving retention in STEM [15,18], and boosting student satisfaction with the course [19]. Considering all 
these benefits, in Fall 2017, the Chemistry Department at the institution where the study took place recruited a group of undergraduates (referred to as Undergraduate Teaching Assistants, UTA), trained them, and introduced to the general chemistry lab sections to assist GTAs in monitoring and guiding students. This pilot program was launched with the goal of providing chemistry students an additional source of information who is a peer, giving GTAs more support in the lab, and helping undergraduate students formulate a deeper understanding of chemistry through teaching. The goal of this research was to examine the effectiveness of the pilot program and determine if the presence of UTAs in lab results in similar findings identified in current studies evaluating peer learning $[17,19]$. In order to achieve this objective, extensive data collection and analysis were conducted as explained in detail in the methodology section.

\section{Methodology}

\subsection{Research Questions}

This study aimed to determine the differences, if any exist, between students' interactions with UTAs and GTAs, and document the methods used by these two groups of teaching assistants to engage the students in chemistry laboratories. The study was guided by two research questions (RQ):

RQ1: How does the extent and type of interactions between UTAs and GTAs in the laboratory section compare?

RQ2: How does the instructional context discussed in these interactions compare for UTAs and GTAs?

\subsection{Participants}

The research was conducted at a large public institution in northern California. For most STEM major requirements, students complete two or three quarters of general chemistry. The instruction of the class has three components: three 50-min lectures, a 50-min discussion, and a 170-min lab. The lab sections are led by a GTA who facilitates the laboratory experiments, acting as the students' main information source throughout the lab. It should be noted that although GTAs participate in a one-and-half day orientation led by the lab coordinator before the quarter begins, they do not get formal training on effective teaching methods. Most of the discussion focuses on practical aspects of running lab and keeping students safe in the lab. A traditional lab approach is used in which students follow step-by-step procedures to set up the experiment, collect data, and draw conclusions. During experiments, dedicated GTAs circulate to answer inquiries or pose guiding questions to students.

In Winter 2017, the students who were registered in the third course of the General Chemistry series were informed about this new UTA program and invited to complete a survey. The survey questions probed students' interest in participating in this program and asked students to describe their skill set. A total of 163 students completed the survey. After evaluating their responses and performances in both lab and lecture portions of the course, 28 undergraduate students were invited to participate. All UTAs completed a course called "Facilitating Learning", traditionally offered to introduce the effective teaching strategies to LAs serving in discussion sessions or lectures. UTAs were also required to complete an online safety course and in-person safety training with the lab coordinator before they joined the labs in Fall 2017. Among these UTAs, only 10 of them agreed to participate in the study and recorded their sessions. Therefore, 10 GTAs were invited to continue the study even though 13 GTAs previously had agreed to join the study.

\subsection{Design}

To obtain a clear picture of the lab instruction and understand the type and scope of interactions, all the conversations between students and undergraduate and graduate teaching assistants were audio-recorded by UTAs and GTAs. Both GTAs and UTAs recorded multiple lab sessions, which resulted in 36 recordings. When comparing UTAs' recordings 
to those of GTAs from the same session, it was noted that some of the recordings did not have equal length. To preserve consistency for data quantity across the sections, the shorter and incomplete recordings were excluded. Moreover, some recordings were determined to have poor audio quality, which made it impossible to transcribe them. Therefore, some recordings and sessions were excluded from the analysis. In the end, only 20 recordings were manually transcribed by the authors of this paper. Out of 20 recordings, 10 came from GTAs' pile and 10 from that of UTAs. Within the completed transcripts, 2-min timestamps were also added to create small intervals to simplify coding as recommended by Velasco and Knedeisen [8]. Then, the same students as a group coded all the transcripts through extensive discussions. The recorded transcripts came from laboratory sections that were jointly staffed by both a UTA and a GTA in order to make qualitative comparisons for the interactions between each type of TA.

\subsection{Data Analysis}

Student interactions with the lab instructors were coded using modified Laboratory Observation Protocol for Undergraduate STEM (m-LOPUS) $[8,20]$. Qualitative data analysis methods were used in lieu of quantitative methodology. Given the small sample size, the statistical power of these findings would be minimal. Future studies will focus on quantitative comparisons.

The original LOPUS codes were modified to better suit this study's purposes and audio data format. Some modifications include excluding codes pertaining to video data and adding some codes to better describe some situations encountered when analyzing the audio data. m-LOPUS has been integrated into Generalized Observation Reflection Platform (GORP) [21]. This web platform permits live or video-based coding (see Figure 1).

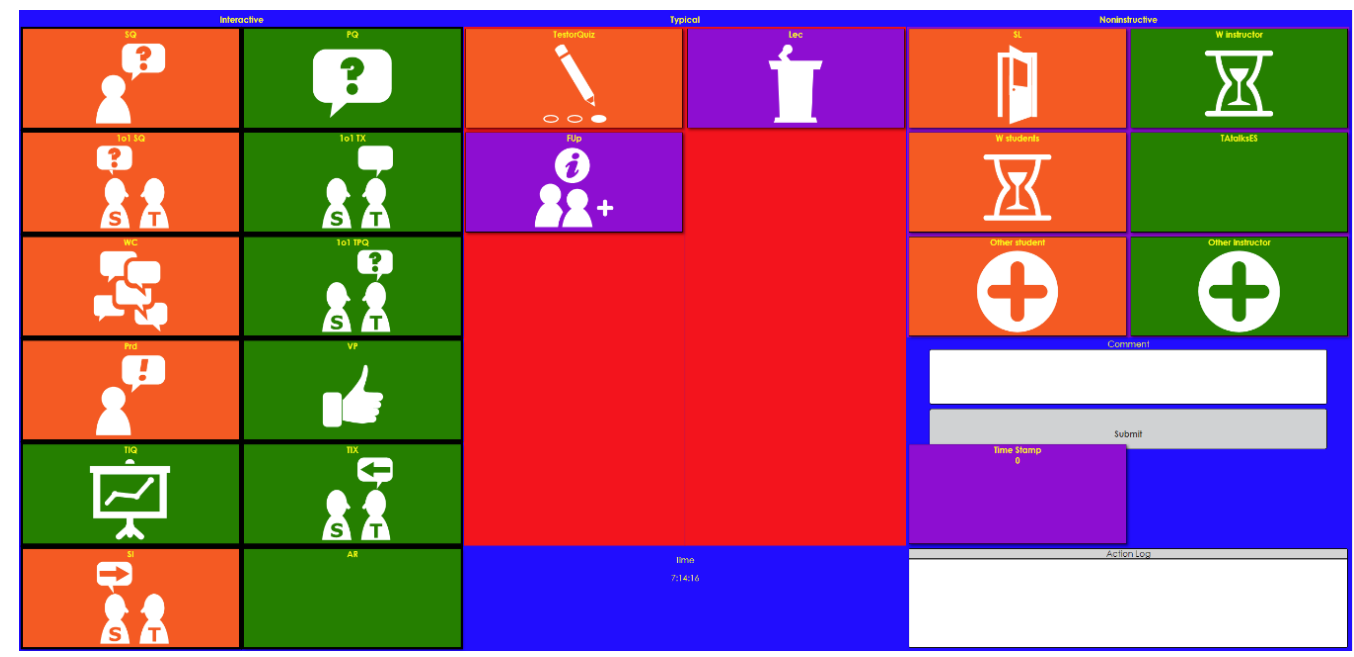

Figure 1. The screenshot of GORP interface with m-LOPUS codes.

GORP also provides a basic analytical report and an Excel spreadsheet for further analysis of the selected codes. GTAs' and UTAs' behavior codes are represented with green or purple buttons, and students' behavior codes are represented with orange buttons. The main codes assigned to statements or questions made by GTAs or UTAs that occur at the beginning of a conversation or within a conversation were summarized in Table 1 (1o1-TX, 1o1-TPQ, TIX, TIQ, VP, AR, Lec, and FUp). On GORP, when one of these codes was selected for the interaction, each code was also coupled with a sub-code to denote the context of the statement or question. These tables also include descriptions of each code and examples of statements or questions from the data transcripts that would receive the designated code. 
Table 1. Descriptions and examples of the m-LOPUS codes $\S$ used to capture GTA and UTA's instructional behaviors.

\begin{tabular}{|c|c|c|}
\hline Code & Description & Example from Transcripts \\
\hline 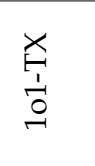 & $\begin{array}{l}\text { GTA/UTA provides a one-on-one explanation to } \\
\text { an individual student or a small group of } \\
\text { students during a conversation. }\end{array}$ & $\begin{array}{l}\text { Student: So why do we make those solutions? } \\
\text { GTA: You will do your Beer's Law Plots with them, so you did part I. You take } \\
\text { your TZ and your AR spectrum and then after that you got one to do your TZ } \\
\text { spectra or TZ absorbance at } 430 \mathrm{~nm} \text {, and then next week you will do. }\end{array}$ \\
\hline 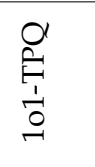 & $\begin{array}{l}\text { GTA/UTA poses a question to an individual } \\
\text { student or a small group of students during } \\
\text { a conversation. }\end{array}$ & $\begin{array}{l}\text { Student: So right here you tell me we didn't have to calibrate again, so } \\
\text { we also didn't calibrate for part } 3 \text {, I guess? } \\
\text { GTA: Do you want a single wavelength part, or you are just collecting? } \\
\text { Student: We are on this. }\end{array}$ \\
\hline 尼 & $\begin{array}{l}\text { GTA/UTA initiates a conversation with an } \\
\text { individual student or a small group of students } \\
\text { with a statement, explanation, or comment. }\end{array}$ & $\begin{array}{l}\text { GTA: If you flip it in another way, it's a lot easier. Flip it. There we go. } \\
\text { Student: Oh, I see }\end{array}$ \\
\hline$\stackrel{O}{O}$ & $\begin{array}{l}\text { GTA/UTA initiates a conversation with an } \\
\text { individual student or a small group of students } \\
\text { with a question. }\end{array}$ & $\begin{array}{l}\text { UTA: Hello!! Just making solution? } \\
\text { Student: Huh? } \\
\text { UTA: making solution? } \\
\text { Student: Yeah. }\end{array}$ \\
\hline$\xi$ & $\begin{array}{l}\text { Verbal monitoring and positive reinforcement by } \\
\text { a GTA/UTA during a conversation. }\end{array}$ & $\begin{array}{c}\text { GTA: Okay, it looks good to me. } \\
\text { Student: Is it really? } \\
\text { GTA: Yeah. So, does that make sense to you guys? Looking at what } \\
\text { color it is absorbed. } \\
\text { Student: Yeah }\end{array}$ \\
\hline$\frac{\approx}{4}$ & $\begin{array}{l}\text { GTA/UTA answers a student's or a small group } \\
\text { of students' questions with a short, direct } \\
\text { response or statement during a conversation. }\end{array}$ & $\begin{array}{l}\text { Student: Should we redo it? } \\
\text { GTA: Yeah, redo the calibration. }\end{array}$ \\
\hline$\stackrel{\circlearrowright}{\unlhd}$ & $\begin{array}{l}\text { GTA is lecturing the class (lab introduction, lab } \\
\text { concepts, safety information, etc.) without } \\
\text { interacting one on one with any student or small } \\
\text { group of students. }\end{array}$ & $\begin{array}{l}\text { GTA: Alright, so in Excel, you are going to plot your one column with your } \\
\text { concentration and one column with your absorbance. In this case, you are } \\
\text { doing Tartrazine at } 430 \mathrm{~nm} \text { and then what you are going to do is you are going } \\
\text { to highlight both columns. You will click chart, scatter, a mark scatter plot, } \\
\text { which should give you this. And then from there, you gonna chart layout... }\end{array}$ \\
\hline 穴 & $\begin{array}{l}\text { GTA/UTA follows up with a student regarding } \\
\text { something discussed in a previous conversation } \\
\text { at the beginning of or during a conversation. }\end{array}$ & $\begin{array}{l}\text { GTA: Is it still not working? } \\
\text { Student: Yes, they cancel the balance. } \\
\text { GTA: That is very unfortunate. I don't know why. Did you try to restart } \\
\text { it, right? So maybe the screen, fix the screen. }\end{array}$ \\
\hline
\end{tabular}

$\S$ Each code was applied to the italicized dialogue in each example.

Table 2 includes the main codes assigned to statements or questions made by students in conversations with a GTA or UTA. Similar to the codes presented in Table 1, when 1o1-SQ and SI codes were assigned, context codes were associated with them as well. The descriptions of each code and examples taken from the data transcripts are presented below.

In order to better understand the context of the codes described in Tables 1 and 2, additional context codes (see Table 3) were assigned. These context codes include Conceptual (Cpt), Analytical (Ana), Experimental (Exp), Safety (Sft), and Miscellaneous (Misc). These context codes provided an additional depth for the comparison of the GTAs' and UTAs' interactions with students in each lab session. In the table below, the descriptions of each context code and the applicable examples for them are provided. 
Table 2. Descriptions and examples of m-LOPUS codes $\S$ used to capture students' interactions with the GTA or UTA.

\begin{tabular}{|c|c|c|}
\hline Code & Description & Example from Transcripts \\
\hline $\begin{array}{l}0 \\
0 \\
1 \\
0 \\
0 \\
0 \\
-1\end{array}$ & $\begin{array}{l}\text { A student or a small group of students asks the } \\
\text { GTA/UTA a question during a conversation }\end{array}$ & $\begin{array}{l}\text { GTA: Take one of these pipettes, and rinse it } \\
\text { Student: Can I pour it down the drain? } \\
\text { GTA: No, it has to go to the cation metal waste. }\end{array}$ \\
\hline ๘ & $\begin{array}{l}\text { A student or a small group of students initiates a } \\
\text { conversation with the GTA/UTA, by asking a } \\
\text { question, making a statement, or there is an } \\
\text { indication of student initiation. }\end{array}$ & $\begin{array}{l}\text { Student: Do you know where is the hydrochloric acid? } \\
\text { UTA: Oh, that's in here. That is this pump. Do you know how to use it? } \\
\text { Student: Yeah, I know. }\end{array}$ \\
\hline
\end{tabular}

$\S$ Each code was applied to the italicized dialogue in each example.

Table 3. Descriptions and examples of the additional context codes $\S$ used to denote the context of each statement or question.

\begin{tabular}{|c|c|c|}
\hline Code & Description & Example from Transcripts \\
\hline Conceptual (Cpt) & $\begin{array}{l}\text { A statement or question that } \\
\text { relates to underlying } \\
\text { scientific principles. }\end{array}$ & $\begin{array}{l}\text { UTA: Oh, so you learn solubility rules later in gen chem. I believe they give } \\
\text { you the solubility rules though on a formula sheet. That's really helpful. } \\
\text { After doing a lot of it, you kind of just know...oh, hydroxide doesn't work } \\
\text { with that. But this isn't an aqueous form so when it's aqueous is it really in } \\
\text { its ionic or is it separated? } \\
\text { Student: It's not a solid. } \\
\text { UTA: Yeah, it's not a solid. You literally have sodium ions and nitrate ions } \\
\text { just floating around in there. }\end{array}$ \\
\hline Analytical (Ana) & $\begin{array}{l}\text { A statement or question that } \\
\text { relates to data analysis } \\
\quad \text { or calculation. }\end{array}$ & $\begin{array}{l}\text { Student: How can we know the mole of this one? } \\
\text { GTA: You will find the moles of that. You know the volume you used. } \\
\text { That's what you can use to find the moles. }\end{array}$ \\
\hline Experimental (Exp) & $\begin{array}{l}\text { A statement or question that } \\
\text { relates to experimental } \\
\text { procedures, equipment, or } \\
\text { laboratory techniques. }\end{array}$ & $\begin{array}{l}\text { Student: do we use the same Erlenmeyer flask for all? } \\
\text { GTA: It does not actually matter what size you use. If you want, you can } \\
\text { use the same one and just wash it out. You can check over by the extra } \\
\text { glassware. The size does not matter. }\end{array}$ \\
\hline Safety (Sft) & $\begin{array}{l}\text { A statement or question that } \\
\text { relates to safety and clean } \\
\text { up procedures. }\end{array}$ & $\begin{array}{l}\text { Student: He said change your gloves after this, right? } \\
\text { UTA: Yeah. Let's go change our gloves because nitric acid is no fun. I } \\
\text { should change my gloves too, I touched your beaker. Just to be safe. }\end{array}$ \\
\hline $\begin{array}{l}\text { Miscellaneous } \\
\text { (Misc.) }\end{array}$ & $\begin{array}{l}\text { A statement or question that } \\
\text { relates to } \\
\text { miscellaneous material. }\end{array}$ & $\begin{array}{l}\text { UTA: So, what are you guys learning right now in chemistry? Where are } \\
\text { you guys at? } \\
\text { Student: Oh, right now balancing the equations, I think is the last gas law. }\end{array}$ \\
\hline
\end{tabular}

$\S$ Each code was applied to the entire dialogue given in the example.

After the application of main and context codes, the findings were analyzed to understand the role of both GTAs and UTAs in lab instruction. The main goals were to determine if students interact with UTAs or GTAs more frequently and whether GTAs or UTAs approach students with thought-provoking questions, which is a method commonly underutilized in science labs [22,23].

\subsection{Personal Attributes of TAs}

Because of the limited number of data points, correlations with personal attributes were not considered. Personal attributes may impact student interaction with a specific UTA or GTA, but these attributes are challenging to measure and control for all variables. Personal attributes include gender and ethnicity, which can be assessed. However, a wider scope of attributes including charisma, looks, perceived sexual orientation, and several others may also impact student interactions. All TAs must pass an English language proficiency test to be a TA. Our research was designed to look broadly at the number and type of interactions students and UTAs or GTAs in the laboratory. Because of the small dataset and the number of permutations possible with student attributes, these factors were not controlled nor analyzed in the research. 


\section{Results and Discussion}

The results are divided into two sections based on the research questions. The first section presents the findings and the relevant discussion related to the interaction differences between UTAs and GTAs with students. The second part, on the other hand, examines the variations and types of content commonly found in interactions that occurred between GTAs or UTAs and the students.

\subsection{Comparing the Extent and Type of Interactions between UTAs and GTAs with Students in} the Labs

In order to analyze the differences in this category, first, the total number of interactions between both groups of TAs and students, regardless of their nature, were compared. The interactions represent instances where one of the m-LOPUS codes was applied. When examined, Figure 2 will highlight some key differences between the interactions of UTAs and GTAs. First, it would be noticed that the interactions with UTAs and GTAs with students show regions of maxima and minima, which is expected as students are completing the laboratory experiment, but the interesting thing is the variation in time at which these peaks were observed. At 20,75, and $120 \mathrm{~min}$, there were notable spikes for interactions with GTAs. Conversely, at 35, 55, and 100 min, students' interactions with UTAs made a peak.

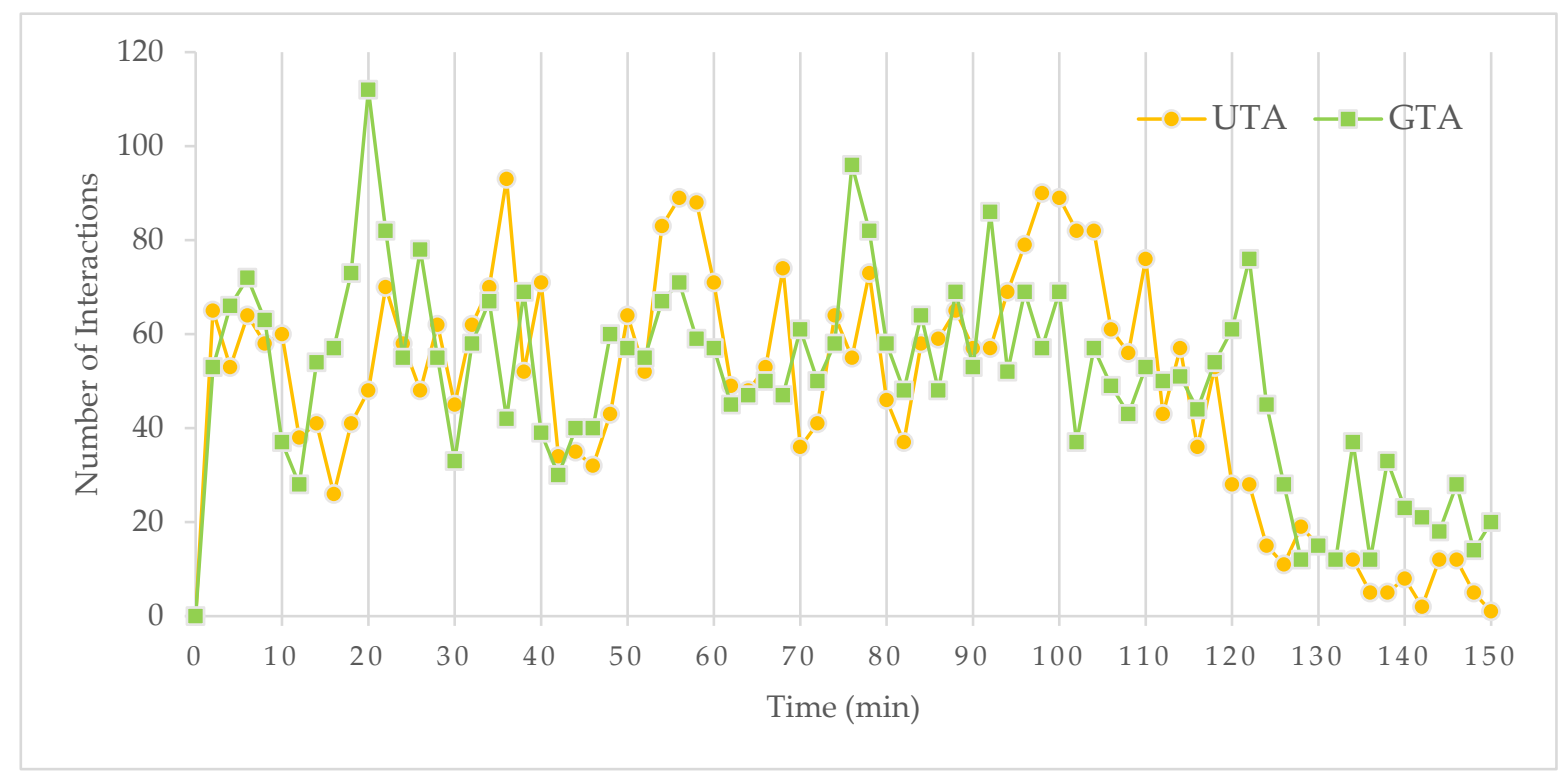

Figure 2. The total number of interactions versus time for GTAs and UTAs.

The relative fewer interactions in the first five minutes indicate that students sometimes waited to engage with the UTA or GTA. The interactions gradually increased and made their first peak around $20 \mathrm{~min}$. The initial maxima observed with GTAs can be rationalized because every laboratory starts with a lecture led by the GTA. The lectures focus on chemistry concepts, techniques, and safety related to the experiment. Students are asking GTAs questions and clarifying content from the pre-lab lecture, which accounts for the first spike.

When GTAs were interacting with a group, the UTAs were instructed to focus on other groups and not interact with that specific group and vice versa. Therefore, only one instructor would interact with a group at a time. As students are working in the laboratory, questions arise regarding procedures, data, and analysis. The spikes represent key time points in the experiment that posed difficulty for students. The second spike arose from questions regarding experimental setup. Questions regarding the setup include: Where can I find the reagents? Where can I find the equipment? Is this the correct setup? Is this the correct glassware? Given the nature of these questions, the UTA likely had greater 
confidence in fielding the questions, and because of the simplicity, the students likely felt more comfortable asking a peer. Around $40 \mathrm{~min}$, the interactions declined. Students successfully setup the experiment and were waiting to collect data. The interactions increased around $55 \mathrm{~min}$ and continued to cycle throughout the rest of the laboratory. At $55 \mathrm{~min}$, students were starting to collect and analyze data and wrap up the laboratory experiment. Therefore, the spikes are associated with questions regarding the validity of the data, the analysis and calculations, disposal, and for notebook signoff. The maxima varied between UTAs and GTAs. All students must have their GTA signoff on their data and notebook, which explains the maxima observed for the GTA close to the 120-min time mark. After $120 \mathrm{~min}$, most students had completed the laboratory, which accounts for the decrease in the number of interactions.

Following an analysis of the total number of interactions, the attention was shifted to the differences in the types of interactions. Figures 3-5 highlight the nature of these differences. Specifically, Figure 3, with three graphs, reveals how the conversations were initiated and by whom. The first graph (Figure 3a) at the top shows differences in the SI code applied. The SI code denotes that the discussion was initiated by students. The middle graph (Figure 3b), on the other hand, shows the number of TIX codes assigned to the interactions over time. The TIX code denotes that the discussion was initiated by UTAs or GTAs providing an explanation to individual or groups of students. The final graph (Figure 3c) highlights the changes observed in the TIQ code during the period of labs. The TIQ code denotes that the discussion was initiated by a UTA or GTA asking a question to individual or groups of students.

As shown in Figure 3a, student-initiated interactions were greater with GTAs. The greater interaction can be attributed to experimental-related questions or questions related to the pre-laboratory lecture. Because the GTAs led the pre-lab lecture at the start of the laboratory, they were viewed as the authority on that specific content and were asked questions either requiring clarification or elaboration.

GTAs have more hands-on laboratory experience than UTAs, which implies they likely feel more comfortable answering questions regarding the procedure, and undergraduates may view them as a greater authority on the content. Several studies support that student view professors and individuals with higher degrees as more knowledgeable [10,24]. Therefore, given the importance of correctly setting up and following key procedures, students may view GTAs as being a greater resource. Students' successful completion of the experiment hinges upon their laboratory technique. Despite the differences observed with regard to the student-initiated questions, the other initiation categories did not have sizable differences. Given the similarities between UTAs and GTAs with the TIX and TIQ codes (see Figure $3 b, c$ ), there is strong support that both types of TAs initiated conversations and assisted with the laboratory. Both demonstrated the initiative to engage with students.

After checking the differences in the initiations of conversations, data were further analyzed to find out who asks more questions, students, UTAs, or GTAs, and to whom. Figure $4 \mathrm{a}, \mathrm{b}$ illustrate the number of TPQ and SQ codes applied to the interactions captured in the transcripts. The code TPQ is associated with instances in which the GTA or UTA posed questions to a student or a small group of students during a conversation. The SQ code is associated with instances in which a student or a group of students posed questions to the GTA or UTA during a conversation. In comparing the results, it was noted that UTAs used questioning strategies more often than GTAs. Specific UTA peaks were observed at 40,60 , and $80 \mathrm{~min}$. This could be the result of the pedagogical course that outlined the best practices for interacting with students in lab and lectures, which is offered to Learning Assistants on the campus. All UTAs were required to take this seminar course before assuming their new role in the lab. Sizable differences were not observed between TIX and TIQ codes above, which supports that both types of TAs engaged with students to a similar extent. The differences observed with the TPQ codes could be attributed to the required course taken by the UTAs, which emphasized strategies for assisting students without directly providing the solution. Therefore, student conversations were initiated 
to a similar extent by UTAs and GTAs, but the nature of the interactions, once they had begun, differed with strategies employed in helping students understand concepts and rationalize solutions.

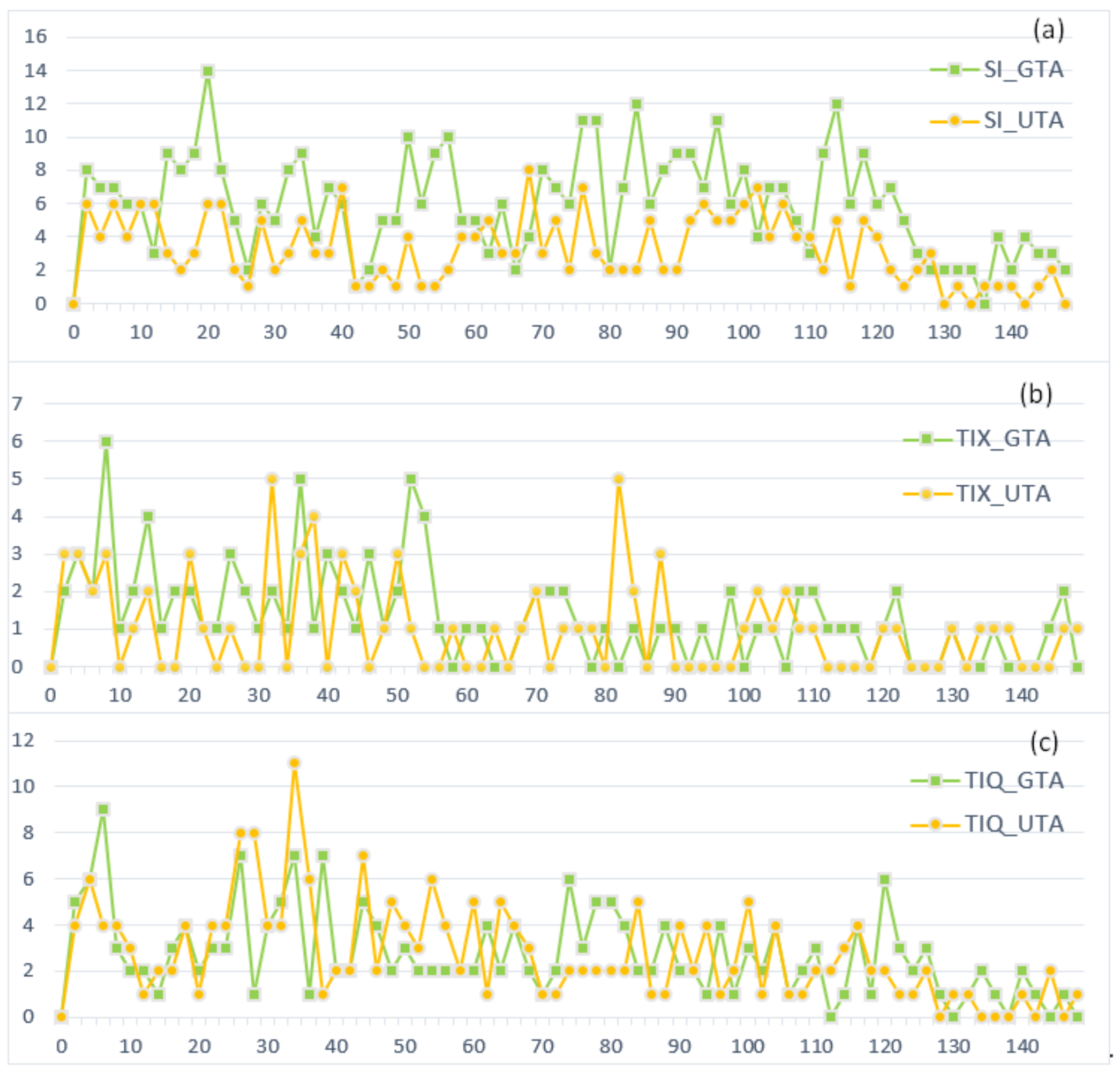

Figure 3. $(\mathbf{a}-\mathbf{c})$ The initiation codes versus time for GTAs and UTAs.

Since GTAs did not take such a course and did not have extensive discussions around these topics, the peaks observed in the UTAs' TPQ codes can be attributed to the training they received in the seminar course. This observation is an important reminder for the lab coordinators and the trainers of teaching assistants that they need to make pedagogical training an integral part of their program to show the GTAs how to effectively guide students to make meaningful connections between theories and the reactions they observe during the experiments, understand the underlying principles, and increase the overall efficiency of lab instruction. The SQ interactions were similar for UTAs and GTAs. The SQ interactions are questions posed once the interaction is initiated. Because of the similarities between the number of SQ interactions with UTAs and GTAs and the continual interactions throughout the laboratory, it can be argued that students again view both UTAs and GTAs 
as valuable resources. Students should be comfortable interacting with and seeking out further clarification from either TA.

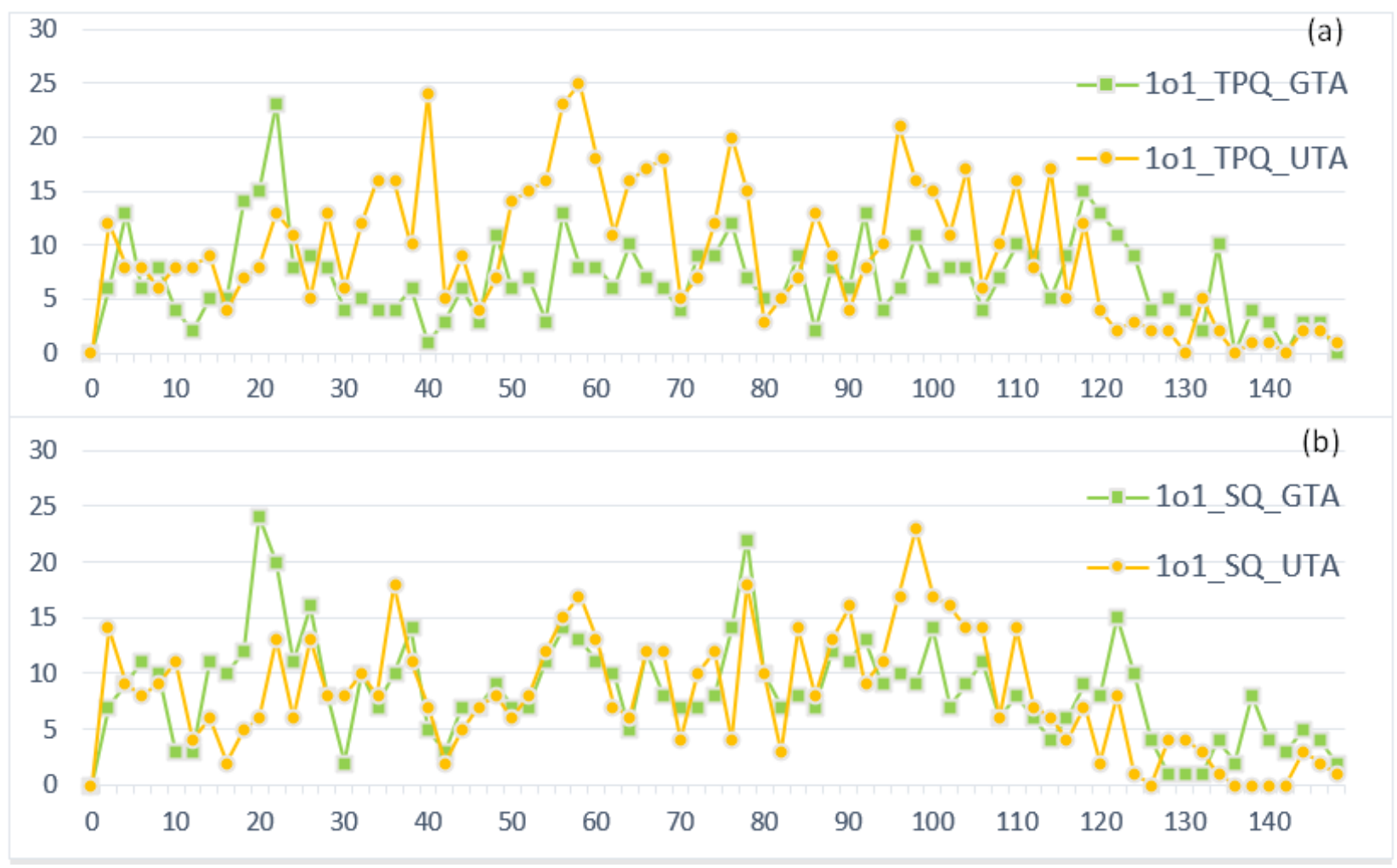

Figure 4. $(\mathbf{a}, \mathbf{b})$ The questioning codes versus time for GTAs and UTAs.

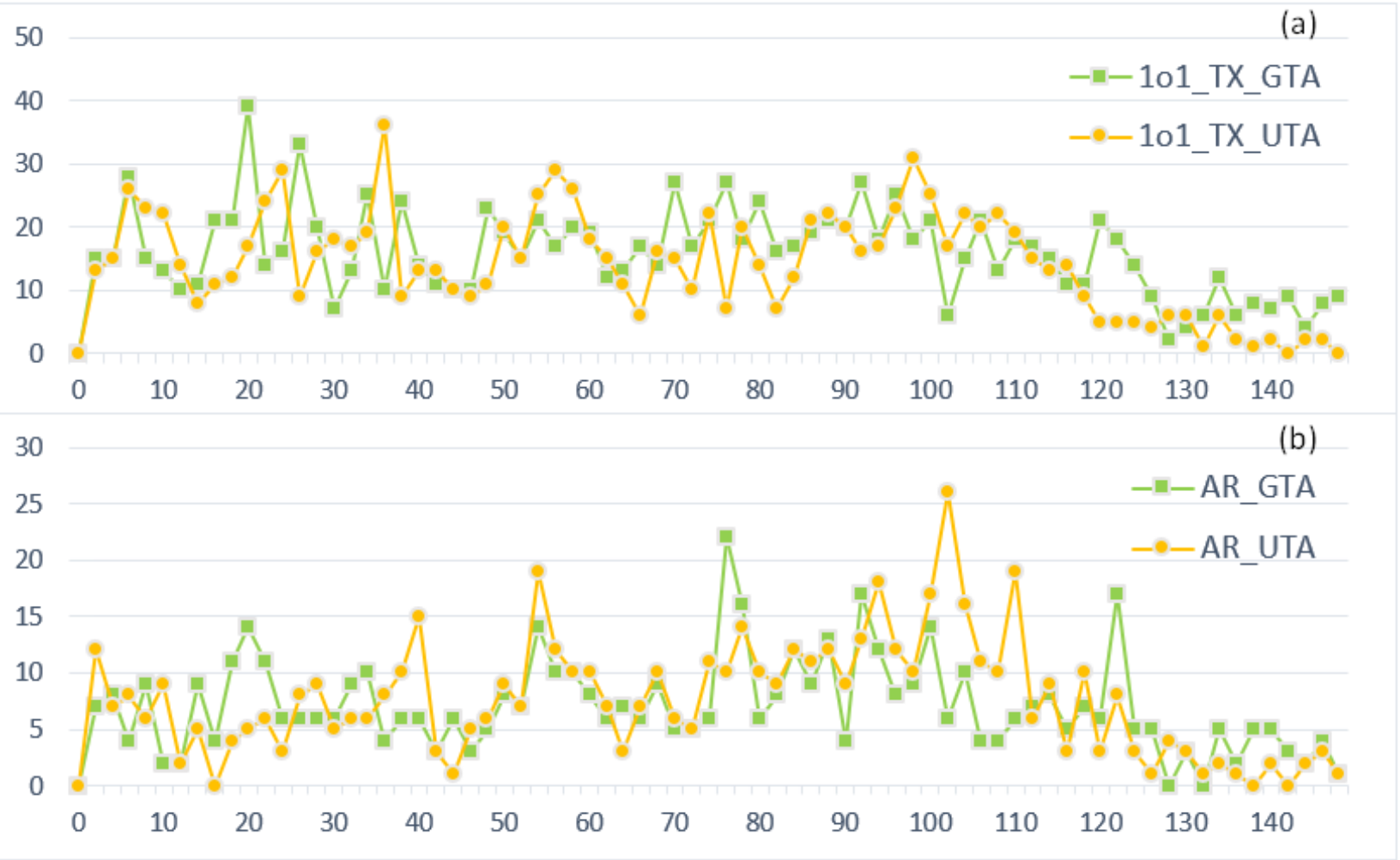

Figure 5. $(\mathbf{a}, \mathbf{b})$. The explanation codes versus time for GTAs and UTA. 
Finally, differences in the nature and frequency of explanations provided by TAs were examined. Figure $5 \mathrm{a}, \mathrm{b}$ show differences for two codes, TX and AR, respectively. The TX code defines instances in which longer, more detailed, explanations were provided by GTAs or UTAs during a conversation. The AR code defines instances in which very short and direct responses were provided by GTAs or UTAs when speaking with students. Sizable differences were not observed in UTAs and GTAs for either explanation code. GTAs and UTAs both used extended responses (TX) and shorter concise responses (AR). This finding supports that both TAs calibrated their responses to the type of question asked. Neither TA used limited or profuse explanations exclusively, which supports their abilities to effectively adapt to the question and students' needs.

In this section, it was determined that although some slight variances existed in initiating conversations, questioning, and explanations of GTAs and UTAs, they do not comprise strong evidence to use against or for either group's superiority in leading discussions in the lab. GTAs are indeed playing an authoritative role in labs, but the extensive interactions between UTAs and students illustrate the importance of having additional support for students in the laboratory. Having UTAs in the labs can help GTAs provide a more effective instruction and mitigate possible injuries and accidents. UTAs can provide this needed support, and the data prove that they are doing a good job compared to their mentors. Furthermore, the majority of GTAs' evaluations that were collected as part of the weekly surveys supported these findings and highlighted the role that UTAs played in the lab. The GTAs were asked to comment on UTAs' effectiveness, quality of interactions with students, and any concerns they had about their instruction in the lab. Although they had the opportunity to inform the instructor about any issues they observed, except very few instances and comments like "she does not ask very probing questions, maybe worried about overstepping my authority though", there were no negative comments on their performance. When survey responses were explored, overwhelming evidence was found that GTAs were very happy with UTAs' presence. One GTA mentioned that "the UTA is good at letting me know when multiple students have similar questions/concerns and is good at notifying me of confusing aspects of the lab that I may assume are easily understood. This allows me to address the confusion quickly". Another GTA praised his UTA partner by pointing out her ability as a teaching assistant and added that "Jenni has been doing a very good job helping students find the answers to their questions without giving the answer directly. She asks questions in order to help the students come to a conclusion." Another positive response made it clear that UTAs were playing a key role in running the labs more smoothly. This GTA particularly indicated that "the UTA does a good job of attending to students when I am currently pre-occupied helping others. Having the UTA available makes the lab go by more quickly and smoothly because our student response time is half of what it would be if I were alone."

\subsection{Analyzing the Context in Interactions between Students and Teaching Assistants}

As discussed in the previous section, an overall examination and quantification of the types of interactions did not reveal many differences between the two TA groups. In this part, the data were scrutinized at a finer level, and the nature of the interactions was explored to reveal any unforeseen differences between the instructional behaviors of the TAs. Figure 6 summarizes the context of the students' discussions with UTAs and GTAs.

Most of the interactions, as shown in Figure 6, focused on experimental aspects of the laboratory. More experimental questions were posed to GTAs than UTAs, but the difference was marginal. While students in the laboratory may have slightly higher confidence in the GTAs' understanding of experimental technique, the data reiterate that UTAs were also viewed as a helpful, reliable source for discussing the experiment. Lack of clarity with the procedure, insufficient laboratory experience for the techniques utilized, or student confidence working in the laboratory are all potential reasons that can account for the number of interactions regarding the experimental procedure. Additional research is 
needed to more closely probe students' experience working in the laboratory to gain a multi-dimensional framework.

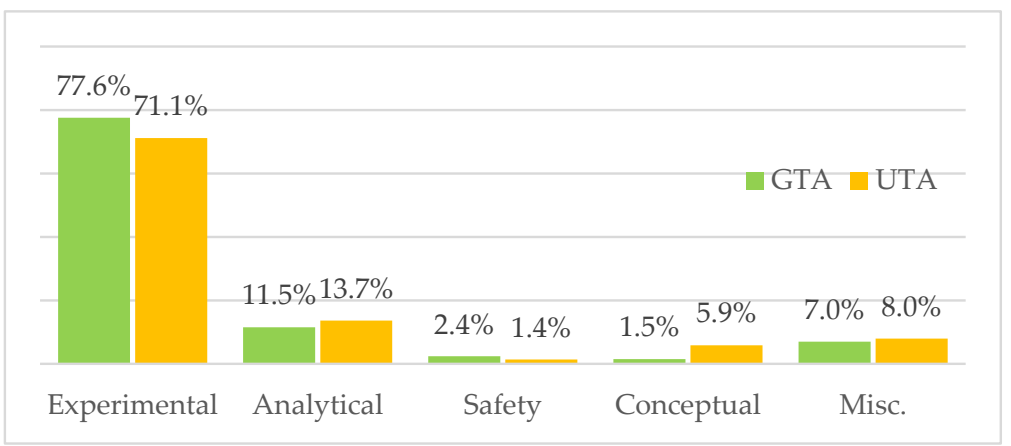

Figure 6. The percentage of contextual codes for GTAs and UTAs.

Interactions regarding analytical, safety, conceptual, and miscellaneous topics were sizably less in comparison to the interactions regarding experimental procedure. The percent communication for the analytical concepts includes data analysis and interpretation. Comparing the interactions in this category reveals that students more frequently interact with UTAs to get help on data analysis strategies and discuss strategies for organizing and writing reports. A similar trend was observed in the conceptual category, which could be explained by the fact that the UTAs were trained to identify questions that probe understanding of concepts. The safety questions were very limited, which is a key point to consider because it illustrates that students either were well informed regarding the safety controls for the experiment or that students were not considering safety hazards to a sizable extent. Future studies will focus on the safety component to identify student perspectives and understanding to further determine why communications regarding safety were very limited. Discussions unrelated to the experiment were categorized as miscellaneous, which included, but were not limited to, discussions about interests, the importance of science, major, and research. These discussions have been attributed to creating an inclusive classroom environment [25].

Figure 7 shows the distribution of all codes observed by their context. Here, it becomes a lot clearer that the "Experimental" context is by far the largest contributor.

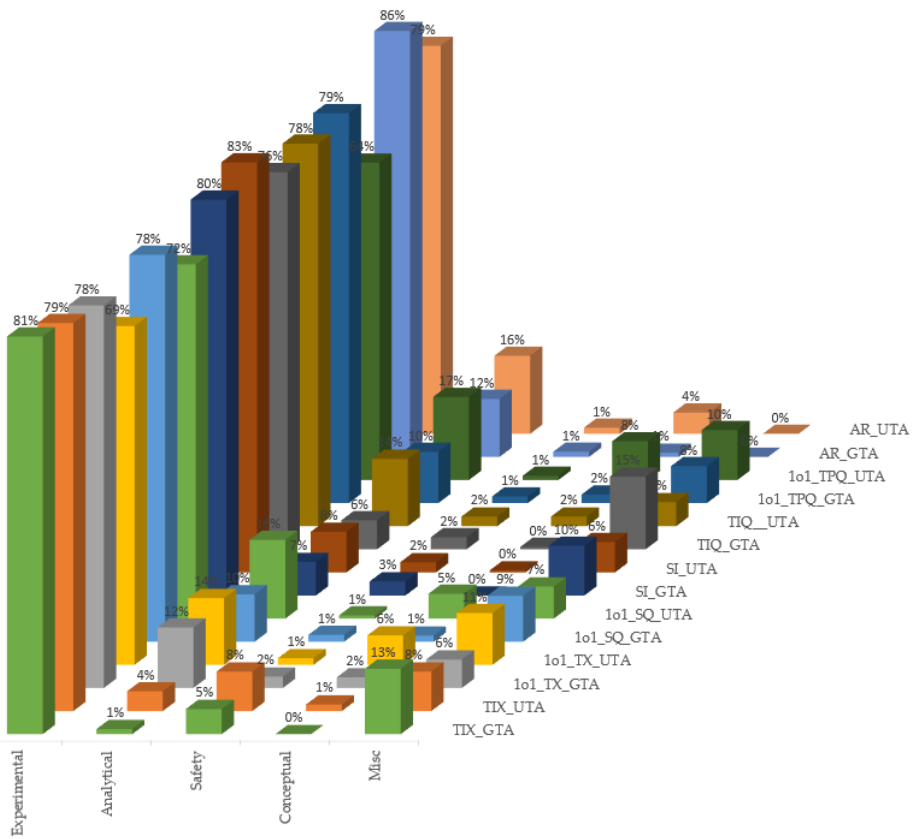

Figure 7. The distribution of codes by content. 
This context is often observed by the researchers who reported a higher frequency of questions regarding the experimental procedure [20]. This category can include questions about how a specific step should be conducted or, more commonly, what the description of a specific step means. The conversations between UTAs or GTAs and students on the lab procedure would often continue at length, and so every interaction in that conversation was recorded as an experimental-context interaction.

It has been observed that the UTAs and GTAs elicit different numbers of interactions in the laboratory with students. Across all contexts with the exception of experimental interactions, UTAs seem to have slightly more interactions than the GTAs did with students, especially in the contexts of analytical and conceptual topics. These findings are consistent with those identified by David, Eric [26]. They reported that students have less anxiety asking their peers questions in lieu of graduate students. The content in the questions provides further support for using UTAs in the laboratory environment. Students sought explanations from both TAs to a similar extent. Given the number of questions, a GTA would likely have been unable to provide the same extent of support needed for students to successfully complete the experiment and analyze the data. Multiple concepts are covered in the laboratory environment including data analysis, extension of concepts from lecture, safety, and laboratory technique. Therefore, the need for additional support in the laboratory environment is not surprising. The questions predominantly focused upon technique, but students did ask a full range of questions to support their understanding.

\section{Limitations}

As mentioned in the participants section, although UTAs and GTAs were able to record 36 sessions, due to unforeseen challenges, 16 recordings were removed from the data analysis due to different length of recordings between UTAs and GTA and poor audio quality. In future studies, a mechanism should be created to remind the TAs to check the battery level before lab sessions start, check the position of the microphone, and the audio quality while recording to prevent such data loss. The resulting smaller data pool hindered our ability to run statistical tests to determine if the differences observed in the interactions between students and GTAs versus UTAs were significant. Video data were originally collected, but the stationary cameras were unable to capture all activity occurring in the lab. Therefore, the limited video data were excluded from the analysis, and this exclusion resulted in the modification of the LOPUS codes to create m-LOPUS.

\section{Conclusions}

These research findings provide support for using UTAs to help supplement GTAs in the laboratory. This conclusion is evidenced by the type and number of interactions observed between students and GTAs versus UTAs as well as by the GTAs' positive feedback on the UTAs' overall performance in laboratory. Because of the similarities in the number of interactions and the content discussed between these two groups, it could be argued that students viewed UTAs as a reliable source of information. There are several comments obtained from the GTAs' weekly evaluation surveys support this claim. In addition to the comments shared earlier, one GTA shared her positive evaluation by indicating that "UTA is good at answering (students') questions, students actually prefer to ask her questions". Another GTA acclaimed his lab partner's contributions by saying that "... she has been doing an awesome job in lab and has been very helpful to me and the students". Although it is impossible to make a strong claim without determining statistical significance, it should still be noted that students had more interactions with UTAs for all topics except for questions on experimental procedure. This is consistent with learning assistant research, which states that students generally feel more comfortable interacting with their peers [27]. Given that GTAs were asked more questions about the experiment, this too is consistent with findings that support students view individuals with higher degrees as having greater authority [10,24]. Therefore, given the importance of understanding the procedure, students likely resonated more strongly with GTAs, thinking 
that GTAs are more influential in their success in laboratory as they were responsible for grading their lab reports. Furthermore, it can be argued that students are more concerned with short-term goals, such as finishing the experiment and getting a good grade on that day, rather than long-term gains, such as developing a strong understanding of concepts [28]. Unlike questions regarding laboratory concepts, questions regarding the procedure are time sensitive and must be addressed in a timely fashion. However, questions regarding concepts, while important, are not time sensitive, and an inaccurate response will not hinder the successful completion of the laboratory but rather the students' long-term understanding of scientific principles. Although students' concern about their grades could be understood to some extent, it is important for science educators to do something about it and use evidence-based strategies to spark an interest in their students. Among successful interventions, introducing topics in context and relating the topics to students' majors, lives, or societies have been widely used and proven to be effective in this regard [29-32]. In such classrooms, it was determined that students become more interested in the topics taught and motivated to do more to be successful [28]. This approach should encourage students not only pay attention to the procedure of the experiments, but also care about the concepts behind the techniques used, be stimulated to connect theory and practice, and see the big picture.

Areas of future research include identifying strategies to better understand the cognitive demand associated with the laboratory setting of chemistry courses. One region of focus is to evaluate whether the current curriculum requires students to do more tasks in lab than feasible for their working memory. Exploring the cognitive demands of the tasks in the lab could also help the instructors decide where to place more emphasis and how to distribute the roles and responsibilities between GTAs and UTAs in lab. It is also important to observe the lab sessions when a single GTA or UTA who has received the same pedagogical training runs the lab and compare the findings to those of this study. A research project in this line would help us better understand how students value their TAs' work and whether the types of interactions show differences in number and nature. In addition, the data on students' learning outcomes should be collected to bring depth to the analysis of the effectiveness of any TA group on students' overall learning gains.

Because of challenges in accounting for all student attributes, metrics such as gender, ethnicity, and other direct or indirect attributes were not included in the analysis. To fully gauge these attributes, additional research with a larger dataset is needed to draw a meaningful conclusion. Additionally, researchers may not be able to catch all attributes that may influence interactions. Future studies will employ quantitative approaches for measuring statistical differences between the student interactions with UTAs and GTAs. Additionally, given the larger dataset, we can implement assessment protocols for measuring the extent of learning that occurs during these interactions. Are these interactions effective and meaningful? Our goal was to analyze qualitatively what is happening in the laboratory with the intent of extending the study to gain a greater understanding of their impact.

Given the limited number of safety-related interactions, more research is also needed to probe whether students understand pertinent safety information or only possess a limited understanding and cannot identify pertinent safety issues to address for the lab. Questions regarding safety can be constructed based upon the hazards associated with reagents and the experimental setup. Future research will address students' understanding of safety protocols and their attitudes regarding safety in the laboratory. Further studies on the teaching effectiveness in the laboratory environment can illuminate inclusive strategies that foster a deeper understanding of chemistry in students.

Author Contributions: Conceptualization, O.G.; Formal analysis, A.T.H.N., X.D.A.-G., M.S., C.T.C.J. and O.G.; Methodology, X.D.A.-G., M.S., L.L.V.T. and O.G.; Writing—original draft, A.T.H.N., C.T.C.J. and O.G. All authors have read and agreed to the published version of the manuscript.

Funding: This research received no external funding. 
Institutional Review Board Statement: The study was conducted according to the guidelines of the Declaration of Helsinki and approved by the Institutional Review Board of University of California, Davis (protocol code is 1130252-1, approved on 17 October 2017).

Informed Consent Statement: Informed consent was obtained from all subjects involved in the study.

Data Availability Statement: The data is available upon request to the corresponding author.

Acknowledgments: The authors would like to acknowledge the efforts of Daniel Erenstein, Jose Sandoval, and Jennifer Kopetzky, former students, who helped with data collection and transcribing of audio recordings.

Conflicts of Interest: The authors declare no conflict of interest.

\section{References}

1. Allen, S.; Peterman, K. Evaluating Informal STEM Education: Issues and Challenges in Context. New Dir. Eval. 2019, 2019, 17-33. [CrossRef]

2. Bruck, L.B.; Towns, M.; Bretz, S.L. Faculty Perspectives of Undergraduate Chemistry Laboratory: Goals and Obstacles to Success J. Chem. Educ. 2010, 87, 1416-1424. [CrossRef]

3. Wheeler, L.; Sturtevant, H.; Mumba, F. Exploratory Study of the Impact of a Teaching Methods Course for International Teaching Assistants in an Inquiry-Based General Chemistry Laboratory. J. Chem. Educ. 2019, 96, 2393. [CrossRef]

4. Deacon, C.; Hajek, A.; Schulz, H. Graduate teaching assistants' perceptions of teaching competencies required for work in undergraduate science labs. Int. J. Sci. Educ. 2017, 39, 2189-2208. [CrossRef]

5. Bretz, S.L. Evidence for the Importance of Laboratory Courses. J. Chem. Educ. 2019, 96, 193-195. [CrossRef]

6. Hofstein, A.; Lunetta, V.N. The laboratory in science education: Foundations for the twenty-first century. Sci. Educ. 2004, 88, 28-54. [CrossRef]

7. Abraham, M.R.; Craolice, M.S.; Graves, A.P.; Aldhamash, A.H.; Kihega, J.G.; Gal, J.G.P.; Varghese, V. The Nature and State of General Chemistry Laboratory Courses Offered by Colleges and Universities in the United States. J. Chem. Educ. 1997, 74, 591. [CrossRef]

8. Velasco, J.B.; Knedeisen, A.; Xue, D.; Vickrey, T.L.; Abebe, M.; Stains, M. Characterizing Instructional Practices in the Laboratory: The Laboratory Observation Protocol for Undergraduate STEM. J. Chem. Educ. 2016, 93, 1191-1203. [CrossRef]

9. Xie, Y.; Fang, M.; Shauman, K. STEM Education. Annu. Rev. Sociol. 2015, 41, 331. [CrossRef] [PubMed]

10. Kendall, K.D.; Schussler, E.E. Does Instructor Type Matter? Undergraduate Student Perception of Graduate Teaching Assistants and Professors. CBE Life Sci. Educ. 2012, 11, 187-199. [CrossRef] [PubMed]

11. Conner, N.W.; Rubenstein, E.D. The Perceptions of the Quality of Education Received from PhD Graduate Teaching Assistant Instructors through the Eyes of Four Agricultural Education Preservice Teachers. NACTA J. 2014, 58, 11-17.

12. Herrington, D.G.; Nakhleh, M.B. What Defines Effective Chemistry Laboratory Instruction? Teaching Assistant and Student Perspectives. J. Chem. Educ. 2003, 80, 1197-1205. [CrossRef]

13. Martin, M.E.; Davidyan, A. Implementing an Undergraduate Learning Assistant Program Tailored for Remote Instruction. J. Microbiol. Biol. Educ. 2021, 22. [CrossRef]

14. Gong, H.J.; Park, H.; Hagood, T.C. Peer learning in STEM: A qualitative study of a student-oriented active learning intervention program. Interact. Learn. Environ. 2020, 1-11, ahead-of-print. [CrossRef]

15. Sellami, N.; Shaked, S.; Laski, F.A.; Eagan, K.M.; Sanders, E.R. Implementation of a learning assistant program improves student performance on higher-order assessments. CBE Life Sci. Educ. 2017, 16, ar62. [CrossRef] [PubMed]

16. Schick, C.P. Trying on Teaching: Transforming STEM Classrooms with a Learning Assistant Program. In Strategies Promoting Success of Two-Year College Students; American Chemical Society: Washington DC, USA, 2018; Volume 1280, pp. 3-27.

17. Van Dusen, B.; Nissen, J.M. Systemic inequities in introductory physics courses: The impacts of learning assistants. arXiv 2017, arXiv:1711.05836.

18. Franklin, S.V.; Hane, E.; Kustusch, M.B.; Ptak, C.; Sayre, E.C. Improving Retention Through Metacognition. J. Coll. Sci. Teach. 2018, 48, 21-27. [CrossRef]

19. Talbot, R.M.; Hartley, L.M.; Marzetta, K.; Wee, B.S. Transforming undergraduate science education with learning assistants: Student satisfaction in large-enrollment courses. J. Coll. Sci. Teach. 2015, 44, 24-30.

20. Wan, T.; Geraets, A.A.; Doty, C.M.; Saitta, E.K.; Chini, J.J. Characterizing science graduate teaching assistants' instructional practices in reformed laboratories and tutorials. Int. J. STEM Educ. 2020, 7, 30. [CrossRef]

21. Tolnay, T.K.; Spiegel, S.; Sherer, J.Z. (Eds.) Development of the Engineering Learning Classroom Observation Tool (ELCOT). In Proceedings of the 2017 ASEE Annual Conference \& Exposition, Columbus, OH, USA, 24-28 June 2017.

22. Cheung, D. Teacher Beliefs about Implementing Guided-Inquiry Laboratory Experiments for Secondary School Chemistry. J. Chem. Educ. 2011, 88, 1462-1468. [CrossRef]

23. Deslauriers, L.; McCarty, L.S.; Miller, K.; Callaghan, K.; Kestin, G. Measuring actual learning versus feeling of learning in response to being actively engaged in the classroom. Proc. Natl. Acad. Sci. USA 2019, 116, 19251-19257. [CrossRef] [PubMed] 
24. Kendall, K.D.; Schussler, E. The Effect of Instructor Title on Student Instructional Expectations. Int. J. Scholarsh. Teach. Learn. 2014, 8. [CrossRef]

25. Cooper, K. Building Student Engagement, Inclusion, and Retention. Danc. Educ. Pract. 2018, 4, 19-24. [CrossRef]

26. David, C.W.; Eric, S.; Ryan, G. Rousing Students' Minds in Postsecondary Mathematics: The Undergraduate Learning Assistant Model. J. Math. Educ. Teach. Coll. 2014, 5. [CrossRef]

27. Hall, S.; Stephens, J.; Andrade, T.; Davids, J.; Powell, M.; Border, S. Perceptions of junior doctors and undergraduate medical students as anatomy teachers: Investigating distance along the near-peer teaching spectrum. Anat. Sci. Educ. 2014, 7, 242-247. [CrossRef]

28. Gulacar, O.; Zowada, C.; Burke, S.; Nabavizadeh, A.; Bernardo, A.; Eilks, I. Integration of a sustainability-oriented socio-scientific issue into the general chemistry curriculum: Examining the effects on student motivation and self-efficacy. Sustain. Chem. Pharm. 2020, 15, 100232. [CrossRef]

29. Zowada, C.; Siol, A.; Gulacar, O.; Eilks, I. Phosphate Recovery as a Topic for Practical and Interdisciplinary Chemistry Learning. J. Chem. Educ. 2019. [CrossRef]

30. Pazicni, S.; Flynn, A.B. Systems Thinking in Chemistry Education: Theoretical Challenges and Opportunities. J. Chem. Educ. 2019, 96, 2752. [CrossRef]

31. Zowada, C.; Gulacar, O.; Eilks, I. Incorporating a Web-Based Hydraulic Fracturing Module in General Chemistry as a SocioScientific Issue That Engages Students. J. Chem. Educ. 2018, 95, 553-559. [CrossRef]

32. Anna, G.; Christian, Z.; Ingo, E.; Ozcan, G. Exploring Chemistry Professors' Methods of Highlighting the Relevancy of Chemistry: Opportunities, Obstacles, and Suggestions to Improve Students' Motivation in Science Classrooms. Educ. Sci. $2021,11,13$. 